\title{
Cost of Rehabilitation Treatment of Patients With Cerebral Palsy in Korea
}

\author{
Seong Woo Kim, $\mathrm{MD}^{1}$, Ha Ra Jeon, $\mathrm{MD}^{1}$, Taemi Youk, $\mathrm{MS}^{2}$, Jiyong Kim, MD, $\mathrm{PhD}^{3}$
}

${ }^{1}$ Department of Physical Medicine and Rehabilitation, National Health Insurance Service Ilsan Hospital, Ilsan; ${ }^{2}$ Department of Statistics, Korea University, Seoul; ${ }^{3}$ Department of Physical Medicine and Rehabilitation, Inje University Ilsan Paik Hospital,

\author{
Ilsan, Korea
}

Objective To investigate rehabilitation treatment cost of patients with cerebral palsy (CP) according to age.

Methods We analyzed the cost of rehabilitation treatment from 2007 to 2013 for patients diagnosed with CP by sourcing data from the National Health Information Database.

Results While the number of recently born children requiring rehabilitation treatment has decreased, the number of patients requiring this treatment in other age groups has gradually increased. In addition, annual physical therapy, occupational therapy, hydrotherapy, and botulinum toxin injection treatment costs per person have increased. On the other hand, the number of orthopedic surgeries and selective dorsal rhizotomy performed has decreased.

Conclusion This study investigated trends in the cost of treatment for patients with CP. This study can be used as a basis to provide treatment support for patients with CP.

Keywords Cerebral palsy, Rehabilitation, Health care costs

\section{INTRODUCTION}

Cerebral palsy (CP) is a neurodevelopmental disorder that affects the movement and posture of a patient due to non-progressive damage of immature brain. It affects patients throughout their life [1]. CP is a complex disorder with various medical problems, including intellectual disability, sensory impairment such as hearing and vision loss, epilepsy, and so on [2,3]. Depending on the severity of $\mathrm{CP}$ and its associated problems, various treatments are needed for each patient. In addition, CP is typically diagnosed in childhood through a patient's growth and development, although several musculoskeletal problems and functional deterioration can also occur. Continuous therapy and management are needed to minimize the disability related to $\mathrm{CP}$ and secondary complications throughout the patient's life. Various therapies for CP have been published [4]. Considerable medical and so-

Received August 12, 2017; Accepted November 23, 2017

Corresponding author: Jiyong Kim

Department of Physical Medicine and Rehabilitation, Inje University Ilsan Paik Hospital, 170 Juhwa-ro, Ilsanseo-gu, Goyang 10380, Korea. Tel: +82-31910-7885, Fax: +82-31-910-7786, E-mail: halwayskim@gmail.com

ORCID: Seong Woo Kim (http://orcid.org/0000-0002-1548-8147); Ha Ra Jeon (http://orcid.org/0000-0002-4234-8086); Taemi Youk (http://orcid. org/0000-0002-4273-3777); Jiyong Kim (http://orcid.org/0000-0003-4693-8400).

(a) This is an open-access article distributed under the terms of the Creative Commons Attribution Non-Commercial License (http://creativecommons.org/ licenses/by-nc/4.0) which permits unrestricted noncommercial use, distribution, and reproduction in any medium, provided the original work is properly cited. Copyright $\odot 2018$ by Korean Academy of Rehabilitation Medicine 
cial costs are needed for these treatments and special care. In the United States, the lifetime cost for patients with CP has been reported to be 11.5 billion US dollars [5]. In Korea, patients are required to be enrolled in the National Health Insurance Service (NHIS). The NHIS is the only public medical insurance institution. It is operated by the Ministry of Health and Welfare in Korea. The National Health Information Database (NHID) is a public database including data on healthcare utilization, health screening, socio-demographic variables, and mortality for the entire population of Korea. It was formed by the NHIS in 2012 [6]. In Korea, healthcare benefits are provided for rehabilitation as well as diagnosis, drugs, treatments, and surgery. Rehabilitation therapy includes physical therapy, occupational therapy, and hydrotherapy, all of which are necessary for patients with CP. Botulinum toxin injection (BTx) for the control of spasticity in CP patients is also covered by NHIS if certain criteria are met. Healthcare services also include non-benefit items. A patient who receives health care treatment pays a certain portion of the healthcare cost as a 'co-payment. The co-payment rate is determined separately from the cost incurred by the inpatient or outpatient service according to the healthcare institution level and type. Typical copayment amounts are $20 \%$ of inpatient hospital care and $30 \%-50 \%$ of outpatient care. Although an option is available to support medical expenses in the case of rare and incurable diseases, it does not apply to CP cases. Thus, families with a CP patient live with a financial burden due to medical expenses incurred during the patient's life. In some other countries such as the United Kingdom and Canada, all medical expenses are funded through the National Health Service [7-9]. Even in some countries such as the United States where private health insurance system is operated, the state is responsible for medical expenses for children with disabilities $[7,10,11]$. In the case of Japan, individuals also pay a certain amount of the entire medical expense while the national health insurance funds the remaining expenses. However, it should be noted that disabled children in Japan are mostly supported by the government in the case of inpatient rehabilitation therapy [12].

The type and amount of management required differ depending on the age of patients with CP. During childhood, management for $\mathrm{CP}$ focuses on achieving maximum development milestones and preventing future complications. In school age and adolescence, treatment for CP needs to prevent musculoskeletal problems and functional deterioration. Even in adulthood, continuous care of these musculoskeletal problems and pain reduction are essential for CP patients. Management of complications that may occur with aging is also necessary. Previous studies have shown that CP patients who are able to walk independently during childhood gradually become non-ambulatory over time due to various problems $[13,14]$.

Therefore, the purpose of this study was to identify rehabilitation treatment cost according to age by using NHID for patients with $\mathrm{CP}$ and investigate the change over time.

\section{MATERIALS AND METHODS}

This study was conducted based on records from the NHID for 7 years from January 1, 2007 to December 31, 2013. We used all records of medical claims for all enrollees. Patients were identified as having CP if their claim records included at least two diagnostic codes according to the Korean Standard Classification of Diseases (KCD). Codes representing CP included spastic CP (G8000, 8001, 8002, 8008, 8009, 8100-8103, 8109-8113, 8119, 8190-8193, 8199, 830-833), dyskinetic CP (G803), ataxic CP (G804), and unspecified (G808). All patients with CP were divided into three groups according to age: $0-6$ years corresponding to infants and toddlers; $7-18$ years corresponding to school age and adolescence; and 19 years or older corresponding to adults. These patients were analyzed based on treatment claim data related to $\mathrm{CP}$.

Costs of rehabilitation therapy including physical therapy, occupational therapy, and hydrotherapy were investigated through NHID data. We analyzed inpatient and outpatient rehabilitation costs separately. Costs of BTx were investigated using corresponding billing procedure codes or corresponding billing drug codes. Costs of orthopedic surgery for correcting musculoskeletal deformity and selective dorsal rhizotomy (SDR) in CP patients were also investigated. Speech and language therapy, cognitive therapy, and the use of an intrathecal baclofen pump were excluded from analysis of treatment costs because they were non-benefit items not supported by NHIS. Costs of assistive devices and appliances for disability were also excluded from the analysis. Insurance- 
covered costs included both insurer's payment and patient's co-payment for medical services. All data were analyzed through Statistical Analysis System (SAS) version 9.4 (SAS Institute, Cary, NC, USA).

\section{RESULTS}

\section{Number of patients with cerebral palsy}

The number of patients with CP increased from 110,557 in 2007 to 130,032 in 2013. By age group, it decreased from 7,401 in 2007 to 5,979 in 2013 for the 0-6 years age group. On the other hand, it increased from 7,293 in 2007 to 8,585 in 2013 for the 7-18 years age group and increased from 95,863 in 2007 to 115,468 in 2013 for the 19 years or older age group.

\section{Rehabilitation therapy of patients with cerebral palsy}

The proportion of patients who had received rehabilitation therapy on admission or on an outpatient basis among all CP patients has increased in recent years. By age group, $76.4 \%$ of all patients with CP in 2007 were treated in the 0-6 years age group. It increased to $77.8 \%$ in 2013. In the case of the 7-18 years age group, this increased from $46.5 \%$ in 2007 to $55.3 \%$ in 2013. For the 19 years or older age group, it increased from $31.5 \%$ in 2007 to $40.5 \%$ in 2013.

The number of patients changed over time. The pattern of change also differed among different age groups. In the 0-6 and 7-18 years age groups, more patients received outpatient rehabilitation therapy than inpatient therapy. However, patients aged 19 years or older were more likely to undergo inpatient rehabilitation therapy than outpatient therapy. In the case of hospitalized patients, the proportion of patients receiving inpatient rehabilitation therapy increased in recent years in all age groups. The proportion of patients in the 0-6 years age group increased from $18.8 \%$ in 2007 to $23.4 \%$ in 2013 . In the 7-18 years age group, the proportion increased from $7.4 \%$ in 2007 to $8.2 \%$ in 2013 . In the 19 years or older age group, it increased from $20.8 \%$ in 2007 to $32.2 \%$ in 2013. The proportion of patients receiving outpatient rehabilitation therapy in the 0-6 years age group was relatively constant. It was $72.8 \%$ in 2007 and $73.1 \%$ in 2013 . The proportion increased from $44.1 \%$ in 2007 to $52.6 \%$ in 2013 in the $7-18$ years age group but decreased from $14.2 \%$ in 2007 to $11.5 \%$ in 2013 in the 19 years or older age group (Fig. 1).

From 2007 to 2013, the total cost of rehabilitation therapy including inpatient and outpatient increased for all age groups. The total cost was significantly higher in the 0-6 years age group and 19 years or older age group than that in the $7-18$ years age group $(p<0.05)$. As of 2013 , the total cost of rehabilitation therapy was KRW 6,229,639 for the $0-6$ years age group, KRW 5,796,557 for the $7-18$ years age group, and KRW 6,462,095 for the 19 years or older age group.

The inpatient rehabilitation therapy cost per person increased 1.9 times from KRW 2,466,698 in 2007 to KRW $4,776,701$ in 2013 for the $0-6$ years age group. For the 7-18 years age group, it increased 2.4 times from KRW $1,587,026$ in 2007 to KRW 3,773,249 in 2013. In the 19 years or older age group, the inpatient rehabilitation

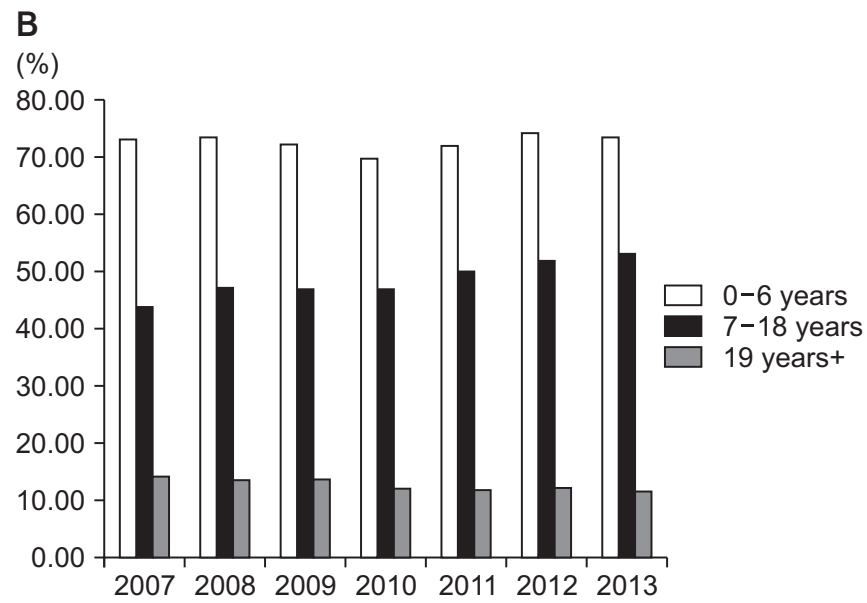

Fig. 1. Proportion of patients receiving inpatient (A) and outpatient (B) rehabilitation therapy. 
therapy cost increased 1.9 times from KRW 2,738,917 in 2007 to KRW 5,232,401 in 2013. In the case of outpatient rehabilitation therapy for the 0-6 years age group, the cost increased 1.2 times from KRW 1,185,188 in 2007 to KRW 1,452,938 in 2013. For the 7-18 years age group, the cost increased 1.5 times from KRW 1,344,596 in 2007 to KRW 2,023,308 in 2013. For the 19 years or older age group, it increased 1.3 times from KRW 930,028 in 2007 to KRW 1,229,694 in 2013 (Fig. 2).

\section{Botulinum toxin injection treatment of cerebral palsy patients}

BTx injection treatment was most frequently performed for the 0-6 years age group. From 2007 to 2013, the proportion of patients undergoing BTx treatment did not change significantly over time in the $0-6$ or 7-18 years age group. However, the proportion increased in the 19 years or older age group (Fig. 3). On the other hand, the injection cost per person increased in all age groups. The cost per person increased 6.7 times from KRW 62,619 in 2007 to KRW 420,687 in 2013 in the 0-6 years age group. For the 7-18 years age group, the cost increased 5.1 times from KRW 59,687 in 2007 to KRW 303,775 in 2013. For the 19 years or older age group, it increased 9.0 times from KRW 62,350 in 2007 to KRW 561,736 in 2103.

\section{Orthopedic surgery for cerebral palsy patients}

Observing orthopedic surgery patterns according to age groups revealed that more orthopedic surgeries were performed for the 7-18 years age group of CP patients. The proportion of patients who underwent orthopedic sur-

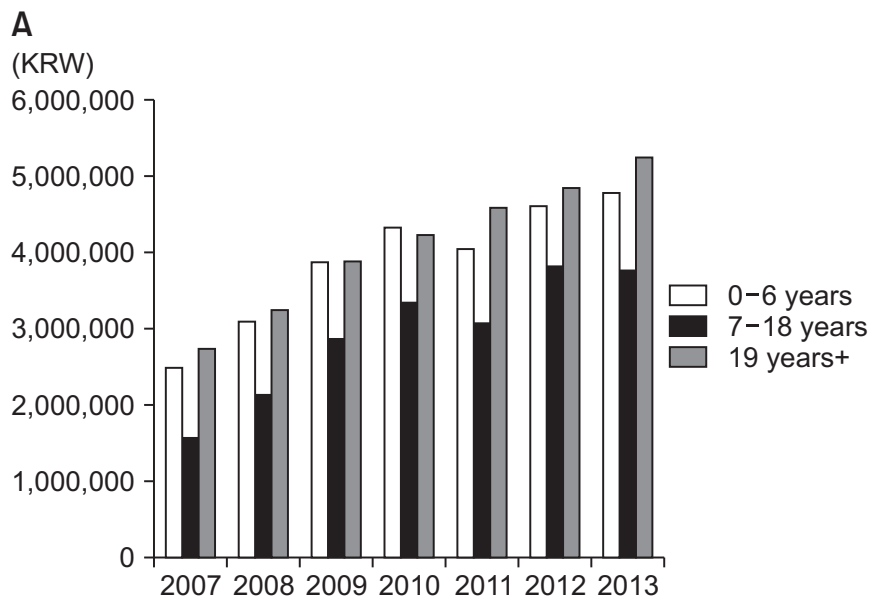

gery among all patients with CP decreased over time for all age groups: from $1.9 \%$ in 2007 to $1.1 \%$ in 2013 for the 0-6 years age group, from $5.7 \%$ in 2007 to $4.0 \%$ in 2013 for the $7-18$ years age group, and from $0.15 \%$ in 2007 to $0.1 \%$ in 2013 for the 19 years or older age group (Fig. 4). The cost of orthopedic surgery was increased rapidly for the 19 years or older age group. The cost of surgery per person was similar from 2007 to 2013 for both 0-6 and 7-18 years age groups However, the cost per person aged 19 years or older increased by 6.3 times from KRW 649,049 in 2007 to KRW 4,081,413 in 2013.

\section{Selective dorsal rhizotomy for cerebral palsy patients}

When we observed SDR data, we found that this operation was performed very rarely in all age groups. It

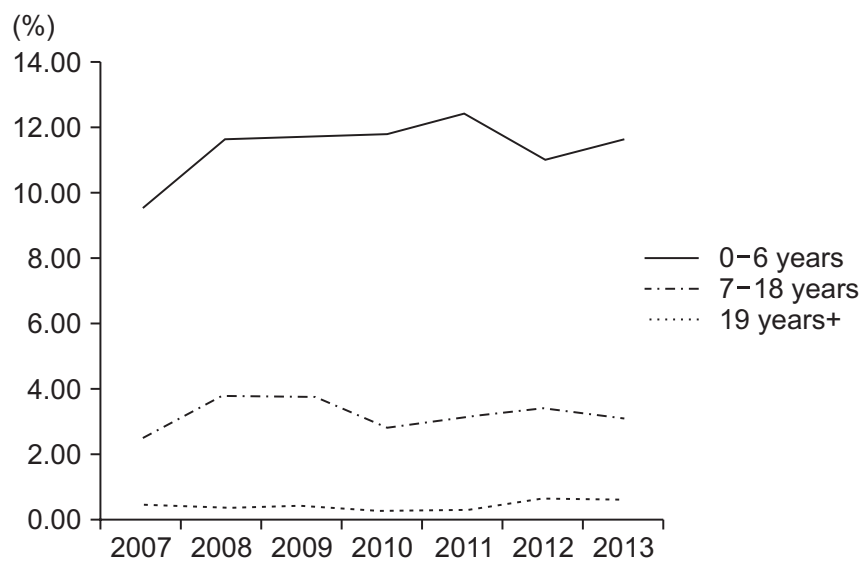

Fig. 3. Proportion of patients administered botulinum toxin injection.

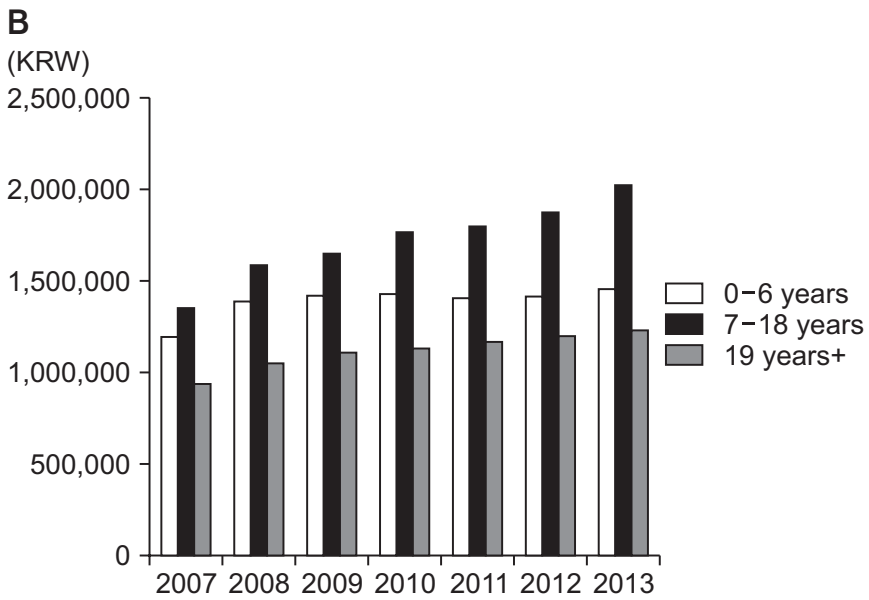

Fig. 2. Cost per person of inpatient (A) and outpatient (B) rehabilitation therapy (unit: KRW). 


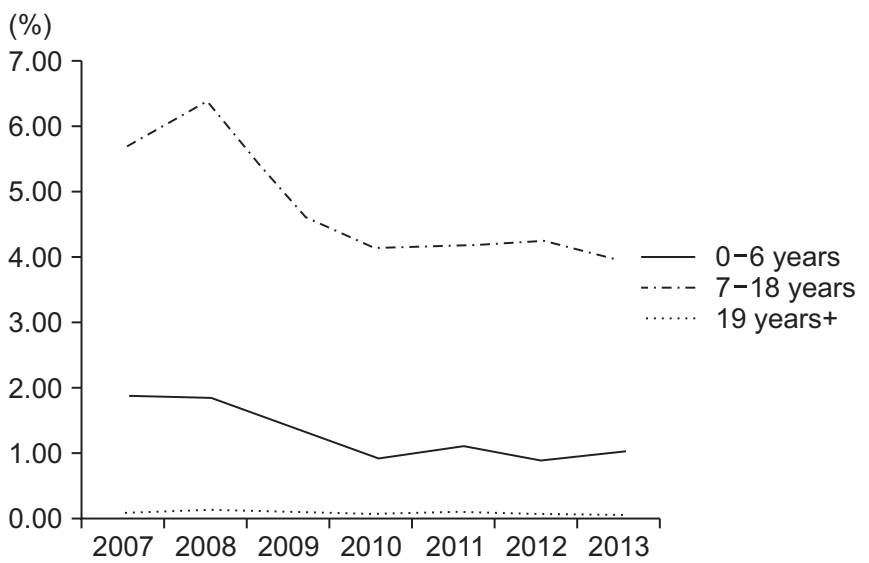

Fig. 4. Proportion of patients undergoing orthopedic surgery.

was most commonly performed for the 7-18 years age group. For the 0-6 years age group, the number of SDRs performed was decreased. The average cost of surgery per person was KRW 338,058-413,190. This has remained constant without any increase or decrease over time from 2007 to 2013.

\section{Total cost of treatment for cerebral palsy patients}

Total cost of treatment per person for each age group combined with rehabilitation therapy, BTx, orthopedic surgery, and SDR is as follows: (1) for the 0-6 years age group, the cost of treatment per person increased 1.7 times from KRW 4,697,811 in 2007 to KRW 8,066,013 in 2013; (2) for the 7-18 years age group, it increased 1.9 times from KRW 4,023,713 in 2007 to KRW 7,448,412 in 2013; (3) for the 19 years or older age group, it increased 2.6 times from KRW 11,518,434 in 2007 to KRW 4,380,344 in 2013. On average, over the 7 years of treatment, expenditure of treatment was higher in the following order: aged 19 years or older $>$ aged 0-6 > aged 7-18 (Fig. 5).

\section{DISCUSSION}

The present study was conducted to investigate rehabilitation treatment expenditures for patients with $\mathrm{CP}$ according to NHID data. Results of this study revealed that annual physical therapy, occupational therapy, hydrotherapy, and botulinum toxin injection cost per person were increased over time. On the other hand, the number of orthopedic surgery and SDR was reduced. The total cost of treatment per person showed a tendency of

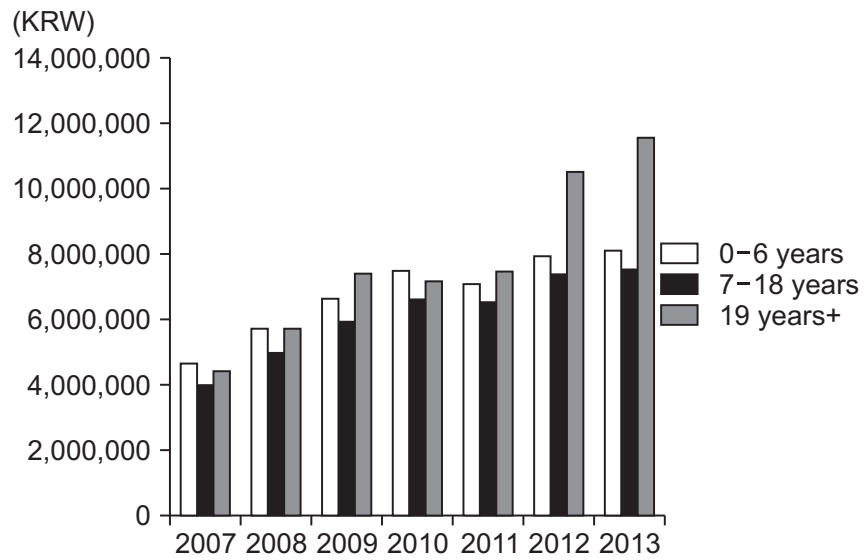

Fig. 5. Total cost of treatment (unit: KRW).

increase for all age groups.

While CP is a disease that occurs in childhood, it affects patients throughout their life. In CP, brain damage is static while functional status and abilities will change. To reduce the rate and prevent these functional impairments, ongoing comprehensive care and management are needed [15]. Conservative and/or surgical treatments of multiple musculoskeletal problems and pain that may occur with age are also required. Results of this study confirmed that many treatment costs might incur even after the patient reached adulthood.

According to the National Statistical Yearbook of National Health Insurance released in 2015, the health expenditure per person according to the age group was about KRW 1,241,778 for 0 -year-old, KRW 1,023,604 for 1-4 years old, KRW 649,854 for 5-9 years old, KRW 417,542 for 10-14 years old, and KRW 400,845 for 15-19 years old. In this study, only CP-related medical expenditure was examined. General medical expenses not associated with CP such as common colds, hypertension, and diabetes mellitus were excluded. Nevertheless, it was found that costs incurred by patients with $\mathrm{CP}$ were significantly more than the average medical expenditure of the entire population. According to unpublished results of work undertaken by the authors, about $25 \%-30 \%$ of children diagnosed with $\mathrm{CP}$ received inpatient rehabilitation treatment while about $60 \%-80 \%$ of them received outpatient rehabilitation treatment. According to a study based on the Korean Database of Cerebral Palsy [16], more than $70 \%$ of children with CP were classified as levels II-V in the Gross Motor Function Classification System. Based on these results, while almost all CP patients should 
have been receiving rehabilitation treatment in childhood, the actual rate of treatment was insufficient. This suggests that rehabilitation costs included in this study might have been underestimated compared to the actual rehabilitation costs required by all CP children, implying more expenditure may be required for proper treatment of CP children. In other countries, according to a report released by Centers for Disease Control and Prevention (CDC), the average lifetime economic cost per CP patient increased from US $\$ 503,000$ in 1992 to US $\$ 921,000$ in $2003[5,17]$. According to a study published in Denmark, the average lifetime economic cost per $\mathrm{CP}$ patient is estimated to be US $\$ 961,000$ [18].

Observation of the pattern of rehabilitation therapy according to age revealed that the 0-6 years age group received more inpatient and outpatient rehabilitation than the 7-18 years age group. Chiarello et al. [19] have reported that younger children receive regular treatment with a frequency of one to five times per week. In contrast, older children receive treatment on an episodic basis according to their specific needs. In the age group of 19 years or older, the proportion of outpatient treatment decreased while the rate of hospitalization increased. This may be because it is difficult to treat CP patients at home after they become adults. In addition, many medical problems can occur. They may require more hospitalization in adulthood than in childhood or adolescence $[15,20]$. In this study, while the analysis did not include treatment cost for general medical problems, the possibility that physical therapy and occupational therapy were performed together during admission due to other medical problems might have some impact on the increment tendency of hospitalization costs in the 19 years or older group.

In Korea, since 2008, the day hospital system was established as a way to provide rehabilitation therapy for children with disabilities. It is a type of medical treatment that is carried out in the hospital for 6 or more hours per day. Patients can participate in various rehabilitation programs and then return home in the evening. For this method, medical expenses are paid based on hospitalization rate. In this study, the increased rate of hospitalization for 0-6 years old children was higher than that for outpatient treatment. It might be partly attributed to the increase in day hospital treatment for intensive rehabilitation.
Insurance coverage for BTx treatment was initiated in 2005. Then in November 2006, the insurance benefit of the motor point block (the injection of a certain drug at a motor point of a muscle) was established. The upper age limit of the insurance policy increased from 5 years old to 7 years old in December 2007. The highest amount of BTx. administered in the 0-6 years age group would be within the limit of the insurance policy describing insurance benefits. Our results showed that the cost of BTx injection was increased more than five times from 2007 to 2013. This seems to be due to an increase in toxin dose per person after insurance coverage had been initiated as well as the policy change over time which provided compensation for injectable needle and cost of procedures.

According to results of this study, orthopedic surgery was performed the most often in the age group of 7-18 years while the total number of orthopedic surgeries decreased each year. A variety of orthopedic surgeries are performed to treat multiple musculoskeletal problems, including joint contracture that can occur in patients with CP. Single-event multilevel surgery (SEMLS) is now considered to be one of the standard treatments to improve gait and function of patients with bilateral spastic $\mathrm{CP}$ whereas single-level surgeries such as isolated gastroc soleus lengthening were performed sequentially over several events in the past $[21,22]$. Effects of SEMLS on patients with CP have been investigated in several studies. The average age of patients undergoing surgery is around 8 to 12 years of age. Results of this study are consistent with results of previous studies showing that orthopedic surgery is performed most frequently in the 7-18 years age group. In young children aged $0-6$, treatment with BTx accounts for a large portion of treatment for spasticity-related problems caused by CP. However, as age increases, the proportion of orthopedic surgery to solve musculoskeletal problems increases.

The number of SDRs performed in patients with CP was low in the total number of cases. This rendered it difficult to accurately analyze the pattern. SDR was advocated by Foerster in 1908. It has been widely used as an effective treatment to reduce lower limb spasticity in patients with CP [23]. However, serious complications related to SDR procedure have been reported and the number of procedures performed has gradually decreased [23]. In recent years, the procedure has been supplemented to increase its safety. It is now being used as a treatment option for 
severe spasticity along with an intrathecal baclofen pump [24].

The total treatment cost associated with $\mathrm{CP}$ has been increasing steadily. This increasing tendency is more pronounced in the 19 years or older group than that in the other two age groups. Especially in the adult group, a large increase is observed in areas of rehabilitation therapy and orthopedic surgery, suggesting that continuous treatment is needed to solve problems such as functional deterioration, pain, and arthritis which may occur in patients with CP during their lifetime.

Only a few studies on the expenditure of medical care for patients with CP have been carried out in Korea. In a study conducted by Park et al. [25], the health care cost of patients with CP was analyzed for 5 years from 2004 to 2008 using data from the Health Insurance Review and Assessment Service (HIRA). The 'healthcare cost' in the study included surgery, medication, physical therapy, orthotics, and hospitalization costs related to CP. The authors concluded that medical expenses related to $\mathrm{CP}$ were higher for younger patients. The lifetime expenditure on CP was about US $\$ 26,383$ per person, which was 1.8 times higher than the medical expenditure per person for non-CP patients at US $\$ 14,579$ [25]. However, the lifetime healthcare cost was sourced from discounted rates, not from actual expenses. Kim et al. [26] have compared treatment expenditures for different ages for children aged 1 to 18 years with CP using data from HIRA. They reported that expenditure on rehabilitation was the highest in childhood, followed by that in preschool, adolescence, and after school age. In the present study, health insurance claim data were used whereas HIRA data were used in previous studies. Thus, analyzed medical expenditure and age groups differed. These results are somewhat difficult to compare directly. However, in both current and previous studies, results showed greater expenditure related to $\mathrm{CP}$ for infants and toddlers than that for school age and adolescence.

This study is meaningful as it is the first study to analyze expenditure for patients with $\mathrm{CP}$ of all age groups using actual claim data. One of the limitations of this study is that only treatment items covered by NHIS could be included in the analysis. Second, the cost of medication, assistive devices, and appliances for disability could not be included. Third, we did not subdivide age groups any further. Lastly, the reliance on diagnostic codes to iden- tify CP might have induced inaccuracy of the results for target patients due to the nature of claims data.

In conclusion, this study investigated the cost of treatment for patients with CP using NHID data. Results demonstrate that annual physical therapy, occupational therapy, hydrotherapy, and BTx costs per person have been increasing rapidly. On the other hand, the number of orthopedic surgeries and SDRs performed has decreased. Results of this study are expected to be used as a basis for establishing insurance policies and support systems for medical expenses of patients with $\mathrm{CP}$ in the future.

\section{CONFLICT OF INTEREST}

No potential conflict of interest relevant to this article was reported.

\section{REFERENCES}

1. Bax M, Goldstein M, Rosenbaum P, Leviton A, Paneth N, Dan B, et al. Proposed definition and classification of cerebral palsy, April 2005. Dev Med Child Neurol 2005;47:571-6.

2. Voorman JM, Dallmeijer AJ, Van Eck M, Schuengel C, Becher JG. Social functioning and communication in children with cerebral palsy: association with disease characteristics and personal and environmental factors. Dev Med Child Neurol 2010;52:441-7.

3. Vos RC, Becher JG, Ketelaar M, Smits DW, Voorman JM, Tan SS, et al. Developmental trajectories of daily activities in children and adolescents with cerebral palsy. Pediatrics 2013;132:e915-23.

4. Novak I, McIntyre S, Morgan C, Campbell L, Dark L, Morton N, et al. A systematic review of interventions for children with cerebral palsy: state of the evidence. Dev Med Child Neurol 2013;55:885-910.

5. Centers for Disease Control and Prevention (CDC). Economic costs associated with mental retardation, cerebral palsy, hearing loss, and vision impairment: United States, 2003. MMWR Morb Mortal Wkly Rep 2004;53:57-9.

6. Seong SC, Kim Y, Khang Y, Park JH, Kang H, Lee H, et al. Data resource profile: the national health information database of the National Health Insurance Service in South Korea. Int J Epidemiol 2017;46:799-800.

7. Doh S, Cole BR. Comparative analysis of health insur- 
ance systems in the United States and South Korea. World Med Health Policy 2009;1:85-116.

8. Steel D, Cylus J. United Kingdom (Scotland): health system review. Health Syst Transit 2012;14:1-150.

9. Hutchison B, Levesque JF, Strumpf E, Coyle N. Primary health care in Canada: systems in motion. Milbank Q 2011;89:256-88.

10. Schoen C, Osborn R, Squires D, Doty MM, Pierson R, Applebaum S. How health insurance design affects access to care and costs, by income, in eleven countries. Health Aff (Millwood) 2010;29:2323-34.

11. Schoen C, Doty MM. Inequities in access to medical care in five countries: findings from the $2001 \mathrm{Com}-$ monwealth Fund International Health Policy Survey. Health Policy 2004;67:309-22.

12. Japan Ministry of Health Labor and Welfare [Internet]. Tokyo: Ministry of Health Labor and Welfare; c2018 [cited 2018 Sep 15]. Available from: http://www.mhlw. go.jp.

13. Andersson C, Mattsson E. Adults with cerebral palsy: a survey describing problems, needs, and resources, with special emphasis on locomotion. Dev Med Child Neurol 2001;43:76-82.

14. Young NL, Gilbert TK, McCormick A, Ayling-Campos A, Boydell K, Law M, et al. Youth and young adults with cerebral palsy: their use of physician and hospital services. Arch Phys Med Rehabil 2007;88:696-702.

15. Gajdosik CG, Cicirello N. Secondary conditions of the musculoskeletal system in adolescents and adults with cerebral palsy. Phys Occup Ther Pediatr 2001;21:49-68.

16. Yim SY, Yang CY, Park JH, Kim MY, Shin YB, Kang EY, et al. Korean database of cerebral palsy: a report on characteristics of cerebral palsy in South Korea. Ann Rehabil Med 2017;41:638-49.

17. Centers for Disease Control and Prevention (CDC). Economic costs of birth defects and cerebral palsy:
United States, 1992. MMWR Morb Mortal Wkly Rep 1995;44:694-9.

18. Kruse M, Michelsen SI, Flachs EM, Bronnum-Hansen H, Madsen M, Uldall P. Lifetime costs of cerebral palsy. Dev Med Child Neurol 2009;51:622-8.

19. Chiarello LA, O'Neil M, Dichter CG, Westcott SL, Orlin M, Marchese VG, et al. Exploring physical therapy clinical decision making for children with spastic diplegia: survey of pediatric practice. Pediatr Phys Ther 2005; 17:46-54.

20. Turk MA. Health, mortality, and wellness issues in adults with cerebral palsy. Dev Med Child Neurol 2009;51 Suppl 4:24-9.

21. McGinley JL, Dobson F, Ganeshalingam R, Shore BJ, Rutz E, Graham HK. Single-event multilevel surgery for children with cerebral palsy: a systematic review. Dev Med Child Neurol 2012;54:117-28.

22. Thomason P, Selber P, Graham HK. Single Event Multilevel Surgery in children with bilateral spastic cerebral palsy: a 5 year prospective cohort study. Gait Posture 2013;37:23-8.

23. Aquilina K, Graham D, Wimalasundera N. Selective dorsal rhizotomy: an old treatment re-emerging. Arch Dis Child 2015;100:798-802.

24. Ingale H, Ughratdar I, Muquit S, Moussa AA, Vloeberghs $\mathrm{MH}$. Selective dorsal rhizotomy as an alternative to intrathecal baclofen pump replacement in GMFCS grades 4 and 5 children. Childs Nerv Syst 2016;32:321-5.

25. Park MS, Kim SJ, Chung CY, Kwon DG, Choi IH, Lee KM. Prevalence and lifetime healthcare cost of cerebral palsy in South Korea. Health Policy 2011;100:2348.

26. Kim DA, Hong HS, Lee HY, Lee HS, Kang MS. Age specificity in general and rehabilitation medical services in children with cerebral palsy. Ann Rehabil Med 2014;38:784-90. 